\title{
Influence of the coefficient of restitution on the classification of granite sand using an elutriator
}

\author{
Eugenia Borsa $^{1 *}$, H. Andrés Petit ${ }^{2}$, Juliana Piña ${ }^{3}$, Cecilia I. Paulo ${ }^{4,5}$, and E. Fabián Irassar $^{4,6}$ \\ ${ }^{1}$ Departamento de Ciencias Básicas, Facultad de Ingeniería, Universidad Nacional del Centro de la Provincia de Buenos Aires \\ (UNCPBA). Av. Del Valle 5737 - 7400 Olavarría -Argentina \\ ${ }^{2}$ Department of Metallurgical and Materials Engineering, Universidade Federal do Rio de Janeiro - COPPE/UFRJ, Cx. Postal 68505, \\ CEP 21941-972 Rio de Janeiro, RJ, Brazil \\ ${ }_{3}^{3}$ Planta Piloto de Ingeniería Química (PLAPIQUI), Universidad Nacional del Sur - CONICET. Camino La Carrindanga km. 7 - 8000 \\ Bahía Blanca - Argentina \\ ${ }^{4}$ Laboratorio de Micropartículas, Centro de Investigaciones en Física e Ingeniería (CIFICEN), dependiente de UNCPBA-CICPBA- \\ CONICET. Av. Del Valle 5737 - 7400 Olavarría -Argentina \\ ${ }^{5}$ Departamento de Ingeniería Química, Facultad de Ingeniería, UNCPBA. Av. Del Valle 5737 - 7400 Olavarría -Argentina \\ ${ }^{6}$ Departamento de Ingeniería Civil, Facultad de Ingeniería, UNCPBA. Av. Del Valle 5737 - 7400 Olavarría -Argentina
}

\begin{abstract}
The objective of this work was to measure the coefficient of restitution of fine granite sand dust (passing sieve $\operatorname{ASTM~N}^{\circ} 30$ ) and to study its influence on the prediction of classification parameters in an elutriator device. Three types of restitution coefficients were evaluated in the computational fluid dynamic model: experimental, elastic, and half the experimental value. The influence of process variables, such as the inclination angle of the equipment and the air inlet velocity, on the efficiency of the obtained fine and coarse fractions was also studied. The results indicate that the coefficient of restitution significantly influences the efficiency parameters calculated for the inclined tests, given the greater contact that exists between the particles and the equipment wall. The experimental value of the coefficient achieved almost total congruence with the coarse fraction collected for the tested velocities. For the vertical tests, the variation of the coefficient did not show significant differences for both collected fractions. The results obtained in this work allowed to gain a deeper insight into the studied gas-solid systems, and to improve the numerical predictions on fine particles classification processes widely used in the ceramic, mining and metallurgical industries.
\end{abstract}

\section{Introduction}

Elutriators are gas-solid separation equipment consisting of one or more vertical tubes, through which a stream of gas contaminated with dust flows upward. Large particles, with terminal settling velocities greater than the upward flow rate of the gas, are separated and collected at the bottom of the device. Smaller particles with low velocities are dragged out of the collector at the top. The size of the particle that can be collected depends on the operating conditions and geometrical dimensions of the separator, but in general it is applied to particles larger than $50 \mu \mathrm{m}$ if the density of the particles is low, and up to $10 \mu \mathrm{m}$ if the particles are relatively dense [1].

The coefficient of restitution is a measure of the energy dissipation between two colliding bodies. It is 0 for perfectly plastic collisions and 1 for perfectly elastic collisions. In CFD simulations it is necessary to specify the values of the tangential and normal CoR of the particle-wall impact. Several studies demonstrate the improvements in the computational fluid dynamics predictions (CFD-DEM) by adjusting the drag coefficient of the particles in the separation processes [2-3]. However, few works report the experimental measurement of the coefficient of restitution for small and irregular particles and its application in improvements to CFD simulations [4-5]

The objective of this work is to measure the coefficient of restitution $(\mathrm{CoR})$ of small and irregular granite sand powder (passing sieve ASTM N ${ }^{\circ} 30$ ) and analyze its influence on the separation efficiency of an elutriator, comparing experimental and numerical results. The influence of other variables of the process such as the degree of inclination of the equipment and the air inlet air velocity is also analyzed and compared with experimental results.

\section{Materials and methods}

\subsection{Coefficient of restitution}

The experimental values of CoR (tangential and normal) were calculated using a previously designed device that was tested by the authors for different types of particles

\footnotetext{
* Corresponding author: eborsa@ fio.unicen.edu.ar

A video is available at https://doi.org/10.48448/5jxb-r970
} 
[4]. The tests were carried out for different impact materials, the values corresponding to iron were used in this work because the elutriator is built with this material. With a high-resolution camera (GoPro Hero 6 Black) the movement of each particle was recorded, in 1080p resolution videos and 240 frames per second (fps). ImageJ 1.5i software was used to determine the velocity before and after the impact needed to calculate the values of CoR. Tangential $\left(\mathrm{CoR}_{\mathrm{T}}\right)$ and normal $\left(\mathrm{CoR}_{\mathrm{N}}\right)$ coefficients of restitution values were calculated for granite sand particles obtained from a quarry in the central area of Buenos Aires's province.

Usually, the coefficient of restitution is defined as [6]:

$$
\text { CoR }=\frac{\text { Vai2 }- \text { Vai1 }}{\text { Vbi1-Vbi2 }},
$$

where $V b i$ and $V a i$ are the velocities before and after the impact of two bodies "1" and "2" that collide. Naming the particle as body "1" and the impact surface as body "2", and considering that in the experimental device the surface is fixed $($ Vbi2 $=$ Vai2 $=0)$, the modulus of the coefficient of restitution $(\mathrm{CoRm})$ is:

$$
\mathrm{CoR}_{m}=\left|\frac{-V a i 1}{V b i 1}\right|=\left|\frac{-V a i}{V b i}\right| \text {. }
$$

To calculate the tangential and normal coefficients of restitution, it is necessary to know the tangential and normal velocity before and after the impact. The detail of the calculations and the used equations was previously reported [4]. Table 1 shows the experimental results of the corresponding CoR values for the studied granite sand particles, the values for elastic collisions $(\mathrm{CoR}=1)$ and half of the values of the experimental $\mathrm{CoR}$, used to compare simulation results. The reported experimental values are the average of 35 tests.

\subsection{Elutriation experiments and simulation}

Figure 1 shows the geometry of the tube-shaped classifier known as elutriator. The particle size distribution (PSD) of the samples fed to the equipment is shown in Figure 2. A total of six cases were performed to analyze the influence of the CoR on the classification performance. The experimental tests were carried out varying the elutriator inclination angle $\left(0\right.$ and $\left.15^{\circ}\right)$ and the air inlet velocity $(1.50,2.25$ and $3.00 \mathrm{~m} / \mathrm{s})$. In all cases the granite sand feed was injected at the top orifice at $3 \cdot 10^{-4} \mathrm{~kg} / \mathrm{s}$. The coarse and fine separation fractions were collected at the bottom and top of the tube, respectively. The PSD of each fraction collected was evaluated by laser granulometry.

CFD simulations of the elutriation experiences were performed using three meshes consisting of 120,000, 200,000 and 400,000 elements. The meshes were evaluated by comparing the pressure loss in the device (Figure 3). As observed, the enhanced wall treatment with the medium mesh gave results in excellent agreement. Further refinement of the mesh did not improve the results. The same evaluation procedure was performed to select the turbulence model (Figure 4). Simulations using the k-e turbulence model (RKE) present better predictions, compared to the SST k-w turbulence model (SST). Particles were injected into the simulation and tracked using the Haider and Levenspiel equation with a measured shape factor of 0.69 . Further details of the numerical simulation implemented can be found in previous studies by the authors [7].

Table 1. Experimental CoR values for granitic sand powder and referential CoR for simulations use.

\begin{tabular}{|c|c|c|c|}
\hline & $\begin{array}{c}\text { CR } \\
\text { (experimental) }\end{array}$ & Elastic & Half \\
\hline $\mathrm{CoR}_{\mathrm{T}}$ & 0.897 & 1 & 0.449 \\
\hline $\mathrm{CoR}_{\mathrm{N}}$ & 0.521 & 1 & 0.261 \\
\hline
\end{tabular}

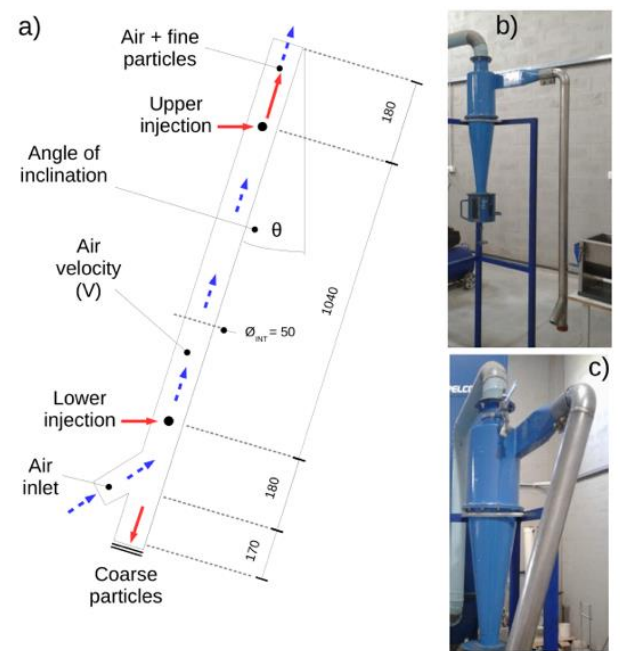

Fig. 1. Layout of the geometry of the tube-shaped classifier. a) CAD modeling. b) y c) Elutriator in pilot plant.

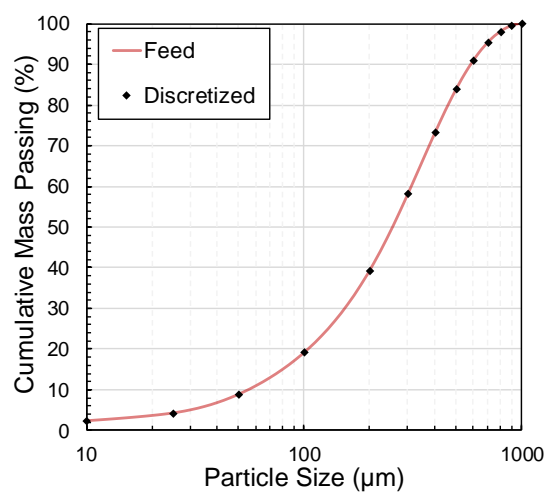

Fig. 2. PSD of the granite sand powder.

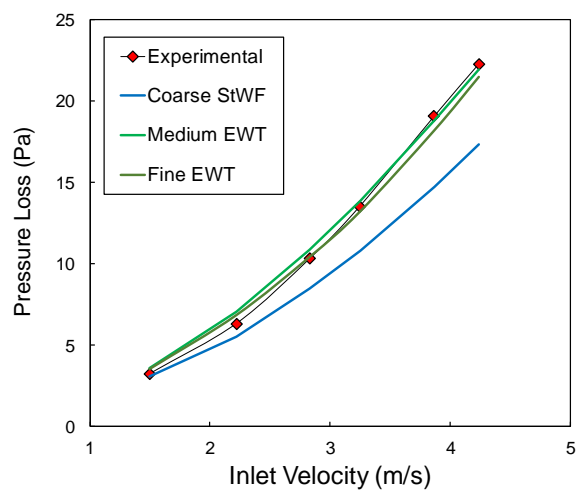

Fig. 3. Pressure loss vs. inlet velocity for different meshes. 


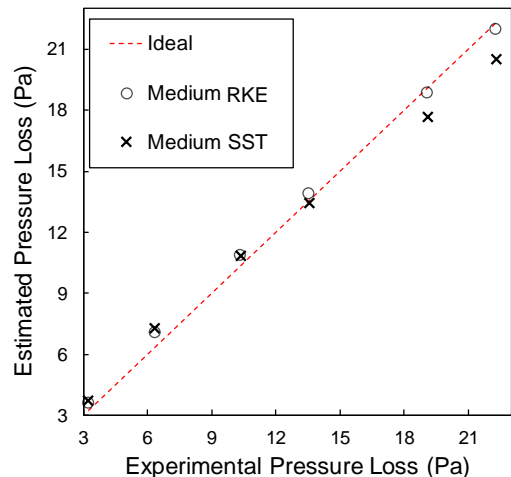

Fig. 4. Estimated pressure loss vs. experimental pressure loss for different turbulence models.

\section{Results and Discussions}

\subsection{Classification efficiency for vertical and inclined tests}

Figure 5 shows the images of the CFD simulation considering the input velocity of $1.5 \mathrm{~m} / \mathrm{s}$ for the vertical test and the three coefficients of restitution tested. It is observed that the coefficient of restitution does not have a great influence on the results, this is because the particles do not interact with the wall to a great extent in the vertical elutriator position $\left(0^{\circ}\right.$ of inclination). Furthermore, the estimation of the classification efficiency improves with the air velocity. This phenomenon is because some clustering, due to adhesion forces in the material, is present and cannot be represented in the simulation model. The adhesion effect is less important at higher air velocities. Figure 6 shows the image of the CFD simulation considering the input velocity of $1.5 \mathrm{~m} / \mathrm{s}$ on the inclined elutriator angle of $15^{\circ}$ and for the three coefficients of restitution tested.

For the inclined cases, the coefficient of restitution has a great influence on the results because particles interact with the walls. In the elastic or high values of CR schemes, particles bounce against the wall and encounter the air. This allows better mixing of the particles with the air during the falling. When the CoR is too low, particles remain attached to the walls and no further classifications occur. This is why the half scheme shows a sharper classification efficiency. That effect also applies to fine particles. As observed in Figure 6, there are no fine particles collected when using the half scheme.

On the other hand, for the elastic and CR schemes, fine particles may have some freedom near the wall without being captured and may roll down the wall taking advantage of the smaller air velocity near the wall. As a consequence, the half scheme gives a coarser cut size while the elastic scheme may represent the fishhook of the classification efficiency effect partially. For higher velocities, (results not shown) the elastic schemes present a sharper classification and the CR scheme presents more mid-sized particles in the collected fraction.

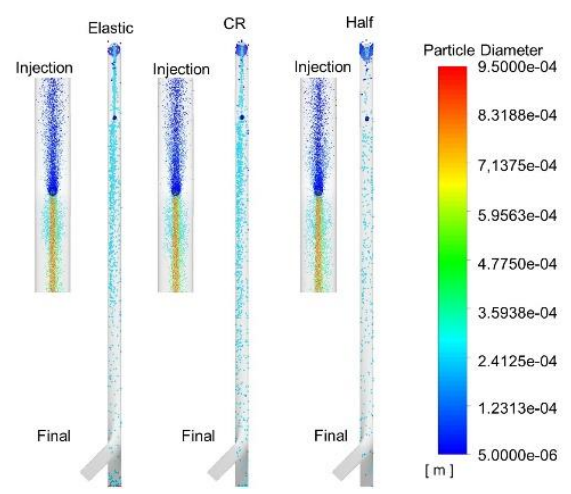

Fig. 5. CFD simulation at $1.5 \mathrm{~m} / \mathrm{s}$ and $0^{\circ}$ of inclination angle, for the three CoR evaluated.

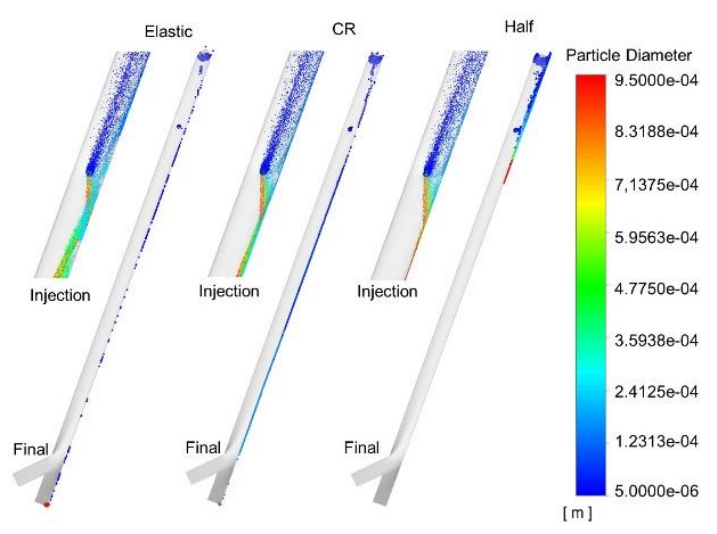

Fig. 6. CFD simulation at $1.5 \mathrm{~m} / \mathrm{s}$ and $15^{\circ}$ of inclination angle, for the three CoR evaluated.

\subsection{Cut size}

Taking the cut size as the main overall parameter, the half scheme presents the worst results. This is observed in Figure 7 where the CFD cut size obtained for each CoR scheme was plotted versus the experimental cut size. The use of the elastic and CR schemes gives differences in the prediction of the classification efficiency, the cut size and the sharpness of cut. The CR controls the freedom of the particles when interacting with the walls and the selection of this value leads to differences in the results.

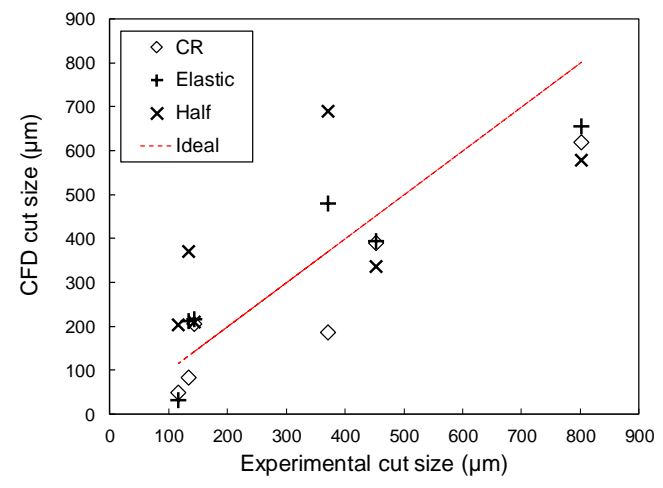

Fig. 7. CFD cut size versus experimental cut size for the CoR evaluated. 


\subsection{Particle size distribution}

Figure 8 shows that the CoR has a small influence on the PSD of the coarse and fine fractions collected for the vertical cases $\left(0^{\circ}\right.$ inclination $)$, for $1.5 \mathrm{~m} / \mathrm{s}$ air velocity. Simulations improve the prediction of the PSD for higher velocities where the effect of clustering and adhesion is lower. The results for the 2.25 and $3.0 \mathrm{~m} / \mathrm{s}$ are not shown but follow the same tendency. The simulated collected fraction is always coarser than the experimental data. As mentioned above, the amount of fines in the collected fraction may be due to the influence of the turbulence, adhesion and inter particles forces. In the case of the half scheme, the PSD of the collected fraction improves the fines content because particles lose momentum with the wall and remain attached (they are not dragged by air).

Figure 9 shows the result for the inclined cases $\left(15^{\circ}\right.$ inclination), for $1.5 \mathrm{~m} / \mathrm{s}$ air velocity. The elastic and CR schemes allow fine particles to roll down the wall and be collected. The PSD of the coarse fraction for these schemes is in better agreement with the experimental data. Similar results have been reported by Liu et al. on the coarse fraction of sand transported in a small pipe [8] although there is not much data on the influence of the CoR on particle classification. For higher velocities, this effect is still present but the air may drag fine particles from the wall. The experimental PSD still presents fines in the collected fraction at high velocities because of the adhesion forces not taken into account in the model. Results for the $2.25 \mathrm{~m} / \mathrm{s}$ are not shown but follow the same tendency. CoR is a necessary parameter for CFD simulations. Although in this work the CoR was calculated as it was defined for spherical particles, for future works the angular rotation at the moment of impact of the particles will be analyzed as it has been done by other authors [9-10].

\section{Conclusions}

The coefficient of restitution of granite sand powder was measure and compared with the value corresponding value to fully elastic particles. The experimental values were used to improve the performance of fluid dynamic simulations on the prediction of classification variables using an elutriator device. The inlet air velocity, the inclination angle of the separator and the coefficient of restitution value affect the classification efficiency, the cut size and the particles size distributions of the obtained products. The numerical results show a great influence of the coefficient of restitution on the separation parameters for the inclined elutriator. The results obtained in this work represent a better understanding of the gas-solid separation processes widely used in the mining, ceramic and metallurgical industries, and a notable improvement in the predictions of computational fluid dynamics models.

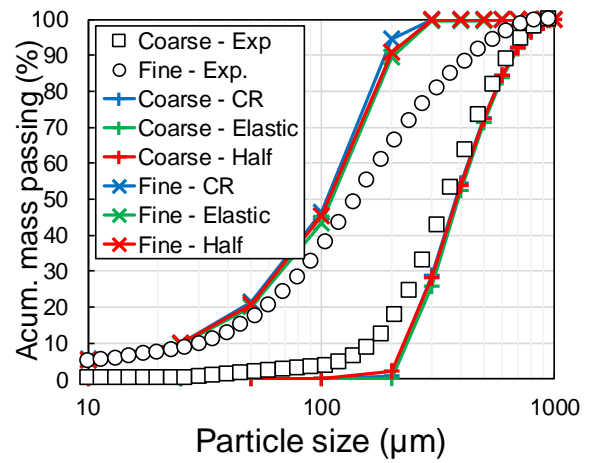

Fig. 8. PSD of products for vertical cases and $1.5 \mathrm{~m} / \mathrm{s}$ varying the CoR.

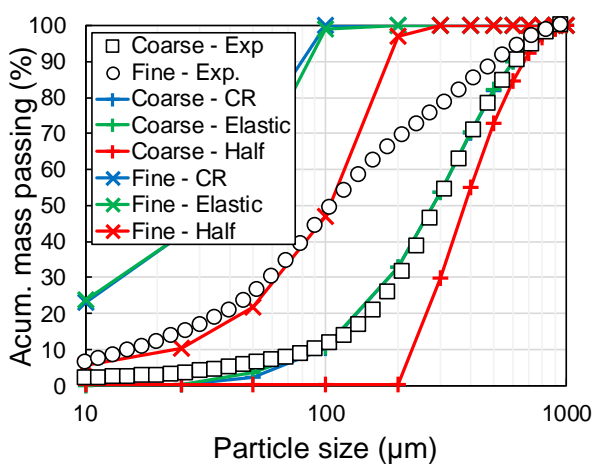

Fig. 9. PSD of products for the $15^{\circ}$ inclined cases and $1.5 \mathrm{~m} / \mathrm{s}$ varying the CoR.

\section{References}

[1] EPA, U.S., Stationary Source Control Techniques Document for Fine Particulate Matter, EPA452/R-97-001 (1998)

[2] N.P. Almeida, M.C. Canhadas, M.R. Medrano Castillo Albertini, K.G. dos Santos J. L. Vieira Neto, Comput Appl Eng Educ. 14 (2020)

[3] C.J. Smith, C.E. Christen, A.J. Andrews, B. A. Olson, C.J. Hogan Jr., J. Aerosol Sci. 146, 105569 (2020)

[4] E. Borsa, C.I. Paulo, H.A. Petit, J. Piña, Lat. Am. Appl. Res. 49, 143 (2019)

[5] C. Lohaa, H. Chattopadhyay, P.K. Chatterjee, Particuology 14, 170 (2014)

[6] R.C. Hibbeler, Engineering Mechanics: Dynamics, (Pearson Prentice Hall, 2010)

[7] H.A. Petit, C.I. Paulo, O.A. Cabrera, E.F. Irassar, Appl. Math. Model. 77, 617 (2020)

[8] W.H. Liu, Y.P. He, F. Wang, M.Z. Li, Effect of Restitution Coefficient on CFD Simulation of Slurry Transport by Pipelines, in Proceedings of the International Ocean and Polar Engineering Conference, ISOPE, 11-16 Oct 2020, Shanghai, China (2020)

[9] A. Glielmo, N. Gunkelmann, T. Pöschel, Phys. Rev. E. 90, 052204 (2014)

[10] P. Müller, D. Krengel, T. Pöschel, Phys. Rev. E. 85, 041306 (2012) 